\title{
DNA damage response-related alterations define the genetic background of patients with chronic lymphocytic leukemia and chromosomal gains
}

\author{
María Hernández-Sánchez ${ }^{\mathrm{a}, \mathrm{b}}$, Ana Eugenia Rodríguez-Vicente ${ }^{\mathrm{a}}$, Isabel González-Gascón y Marín ${ }^{\mathrm{c}}$, \\ Miguel Quijada-Álamo a , Jesús María Hernández-Sánchez ${ }^{a}$, Marta Martín-Izquierdo ${ }^{a}$, \\ José Ángel Hernández-Rivas c, Rocío Benito ${ }^{\mathrm{a}}$, and Jesús María Hernández-Rivas ${ }^{\mathrm{a}, \mathrm{d}}$ \\ ${ }^{\mathrm{a}}$ Servicio de Hematología, IBSAL, IBMCC, CIC Universidad de Salamanca-CSIC, Hospital Universitario, Salamanca, Spain; ${ }^{\mathrm{b}}$ Department of \\ Medical Oncology, Dana Farber Cancer Institute, Boston, Massachusetts, USA; ' Servicio de Hematología, Hospital Universitario Infanta \\ Leonor, Universidad Complutense de Madrid, Madrid, Spain; ${ }^{\mathrm{d}}$ Departamento de Medicina, Universidad de Salamanca, Salamanca, Spain
}

(Received 20 November 2018; revised 24 January 2019; accepted 14 February 2019)

\begin{abstract}
The presence of chromosomal gains other than trisomy 12 suggesting a hyperdiploid karyotype is extremely rare in chronic lymphocytic leukemia (CLL) and is associated with a dismal prognosis. However, the genetic mechanisms and mutational background of these patients have not been fully explored. To improve our understanding of the genetic underpinnings of this subgroup of CLL, seven CLL patients with several chromosomal gains were sequenced using a next-generation sequencing (NGS)-targeted approach. The mutational status of 54 genes was evaluated using a custom-designed gene panel including recurrent mutated genes observed in CLL and widely associated with CLL pathogenesis. A total of 21 mutations were detected; TP53 (42.8\%), ATM (28.5\%), SF3B1 $(28.5 \%)$, and $B R A F(28.5 \%)$ were the most recurrently mutated genes. Of these mutations, $61.9 \%$ were detected in genes previously associated with a poor prognosis in CLL. Interestingly, five of the seven patients exhibited alterations in TP53 or ATM (deletion and/or mutation), genes involved in the DNA damage response (DDR), which could be related to a high genetic instability in this subgroup of patients. In conclusion, CLL patients with several chromosomal gains exhibit high genetic instability, with mutations in CLL driver genes and high-risk genetic alterations involving ATM and/or TP53 genes. () 2019 Published by Elsevier Inc. on behalf of ISEH - Society for Hematology and Stem Cells.
\end{abstract}

Hyperdiploidy is a common cytogenetic abnormality in $\mathrm{B}$ acute lymphoblastic leukemia (B-ALL) and multiple myeloma (MM) and is associated with a good disease course in both hematological malignancies [1,2]. By contrast, hyperdiploidy is a very rare event in chronic lymphocytic leukemia (CLL), occurring at a frequency lower than $1 \%$ and being associated with a poor prognosis [3-5]. Most patients with

Offprint requests to: Jesús-María Hernández-Rivas, IBMCC, CIC Universidad de Salamanca-CSIC, Hospital Universitario de Salamanca, Paseo de San Vicente, s/n 37007 Salamanca, Spain; E-mail: jmhr@usal.es

Supplementary material associated with this article can be found in the online version at https://doi.org/10.1016/j.exphem.2019.02.003. hyperdiploidy required a significantly shorter time to first treatment and had shorter overall survival [4].

It has been suggested that hyperdiploidy is explained by the presence of genomic instability, which could be associated with TP53 disruption [6]. In fact, deletion of 17p (17p -) is the most frequent cytogenetic abnormality concurring with hyperdiploidy in CLL [4]. The implementation of nextgeneration sequencing (NGS) has provided new insights into CLL complexity, identifying a growing list of putative driver genes that underlie the heterogeneity in CLL $[7,8]$. However, little is known about the genetic background underlying hyperdiploidy. Therefore, in this study, we analyzed the mutational status of a panel of CLL mutated genes in a subset of well-characterized CLL patients with hyperdiploidy to 
describe the mutational profile of this infrequent and poorly documented subgroup of CLLs.

\section{Methods}

A total of seven patients with hyperdiploidy were included in the sequencing study. Clinical data were previously reported [4]. The main clinical and biological features of each patient are summarized in Supplementary Table E1 (online only, available at www.exphem. org). The study was approved by the ethics committee of Hospital Universitario de Salamanca. For all samples, written informed consent approved by the ethics committees of the respective institutions were available in accordance with the Declaration of Helsinki. Hyperdiploidy was considered when a gain of at least three of the five fluorescence in situ hybridization (FISH) probes used (11q22/ ATM, 12q13, 13q34, 14q34/IGH, and 17p13/TP53) was observed, as previously described [4]. Five samples were obtained at the time of diagnosis (P3-P7), with the exception of two analyzed at the time of progression (P1 and P2). Genomic DNA was isolated from peripheral blood and/or bone marrow samples using a QIAamp DNA Mini Kit according to the manufacturer's protocol (Qiagen, Valencia, CA, USA). DNA concentration was measured with a Qubit 2.0 fluorometer (Life Technologies, Carlsbad, CA, USA).

Agilent SureDesign was used to perform a design with 8,951 probes (416.393 kbp) targeting 682 regions of a panel of 54 genes previously reported as mutated in CLL or involved in the disease pathogenesis (Supplementary Table E2, online only, available at www.exphem.org) [7,8]. Sequence data generated by the MiSeq platform were analyzed by an in-house bioinformatic pipeline mapping reads to the Reference Human Genome (hg19). The Burrows-Wheeler Aligner (BWA) and Genome Analysis Tool Kit (GATK) were used for variant calling of single-nucleotide variants (SNVs) and short insertions/deletions (InDels) [9,10]. A mean depth of $284 \mathrm{reads} / \mathrm{base}$ within the regions of interest was obtained, with $95 \%$ of regions sufficiently covered $(>100 \times)$. Supplementary Table
E3 (online only, available at www.exphem.org) outlines the coverage data for each sample. Variants were then filtered according to the severity of the consequence, to accept variants leading to an amino acid change in the protein sequence (missense, nonsense, frameshift) and those in the splice site. Minor allelic frequencies (MAFs) were consulted in the Exome Variant Server, 1000 Genomes Browser, and exome aggregation consortium (ExAC) databases, removing those with a MAF $>1 \%$. All accepted mutations were reviewed individually with Integrative Genomics Viewer (IGV, Broad Institute, Cambridge, MA, USA) software.

In line with the sequencing approach explained previously, the mutational status of a cohort of unselected CLL patients without hyperdiploidy was also assessed $(n=50)$. Clinical and biological data from this control group are summarized in Supplementary Table E4 (online only, available at www.exphem.org). Samples were sequenced at the moment of diagnosis or pretreatment.

Statistical analyses were performed using IBM SPSS for Windows, Version 22.0 (IBM Corp., Armonk, NY, USA). The $\chi^{2}$ test was used to assess associations between categorical variables; continuous variables were analyzed with the Mann-Whitney $U$ test. Results were considered statistically significant at $P$ values $<0.05$.

\section{Results and Discussion}

Patients with hyperdiploidy had a higher percentage of CLL driver mutations

Within patients with hyperdiploidy, a total of 21 mutations were detected: 18 missense, 3 stop nonsense, 1 frameshift, and 1 splicing (Table 1; Supplementary Table E5, online only, available at www.exphem.org). The most frequently mutated genes were TP53 (3/7, 42.8\%), ATM (2/7, 28.5\%), SF3B1 $(2 / 7,28.5 \%)$, and $B R A F(2 / 7,28.5 \%)$ (Figure 1A).

Table 1. Summary of the mutations identified by NGS in CLL patients with hyperdiploidy.

\begin{tabular}{|c|c|c|c|c|c|c|c|c|}
\hline Patient ID & Gene & Transcript & $\begin{array}{l}\text { cDNA } \\
\text { change }\end{array}$ & $\begin{array}{l}\text { Protein } \\
\text { change }\end{array}$ & Frequency $(\%)$ & $\begin{array}{l}\text { Depth } \\
(>\text { Q30) }\end{array}$ & COSMIC ID & $\begin{array}{l}\text { Previously described } \\
\text { in lymphoid tissue }\end{array}$ \\
\hline 1 & $A T M$ & NM_000051 & c.C1810T & p.P604S & 54.05 & 74 & COSM22499 & Yes \\
\hline 1 & $B R A F$ & NM_004333 & c.A1781G & p.D594G & 30.63 & 161 & COSM467 & Yes \\
\hline 2 & $M G A$ & NM_001080541 & c.C6217T & p. $Q 2073 X$ & 33.85 & 130 & - & - \\
\hline 2 & TP53 & NM_000546 & c. $451 C>G$ & p.P190A & 33.33 & 111 & COSM99680 & No \\
\hline 2 & RPS15 & NM_001018 & c.C413T & p.S138F & 42.55 & 94 & - & - \\
\hline 2 & $S F 3 B 1$ & NM_012433 & c.A1997C & p.K666T & 4.67 & 107 & COSM131556 & Yes \\
\hline 2 & $S F 3 B 1$ & NM_012433 & c.A1996C & p.K666Q & 11.82 & 110 & COSM132950 & Yes \\
\hline 2 & NOTCH1 & NM_017617 & c.7541_7542del & p.P2514fs & 35.8 & 243 & COSM12774 & Yes \\
\hline 3 & TP53 & NM_000546 & c. $919+1 \mathrm{G}>\mathrm{T}$ & - & 75.21 & 117 & COSM3378337 & No \\
\hline 3 & PCDH10 & NM_032961 & c.A2914G & p.N972D & 45.37 & 205 & - & - \\
\hline 5 & $F U B P 1$ & NM_003902 & c.A1040G & $p \cdot Q 347 R$ & 30.54 & 167 & - & - \\
\hline 5 & $A T M$ & NM_000051 & c. G8440T & p.E2814X & 62.07 & 58 & - & - \\
\hline 5 & $A T M$ & NM_000051 & c. $G 8773 A$ & p.G2925S & 20 & 145 & - & - \\
\hline 5 & $B R A F$ & NM_004333 & c. G1780A & p.D594N & 13.73 & 153 & COSM27639 & yes \\
\hline 6 & IGLL5 & NM_001178126 & c. $C 85 G$ & p.L29V & 13.56 & 317 & - & - \\
\hline 6 & IGLL5 & NM_001256296 & c. $G 21 A$ & p.W7X & 13.13 & 297 & COSM3357309 & yes \\
\hline 6 & $I G L L 5$ & NM_001178126 & c.A146C & p.D49A & 11.9 & 294 & - & - \\
\hline 6 & KLHL6 & NM_130446 & c.T269C & p.L90P & 12.84 & 148 & - & - \\
\hline 6 & KLHL6 & NM_130446 & c.C268T & p.L90F & 12 & 150 & - & - \\
\hline 7 & TP53 & NM_000546 & c.T613G & p.Y205D & 92.5 & 200 & - & - \\
\hline 7 & $S F 3 B 1$ & NM_012433 & c. $G 1874 T$ & p.R625L & 40.76 & 157 & COSM110695 & yes \\
\hline
\end{tabular}


A

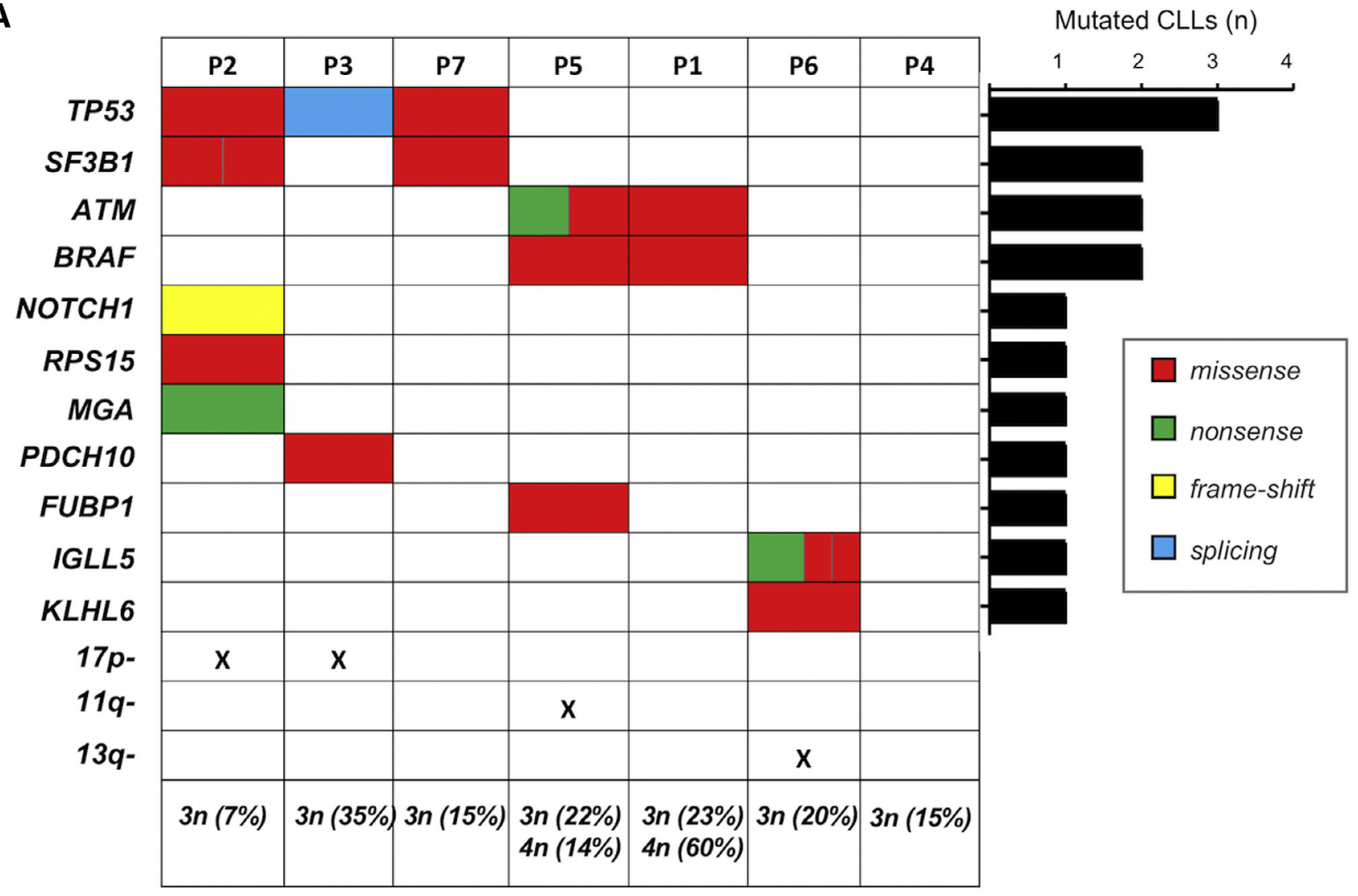

B

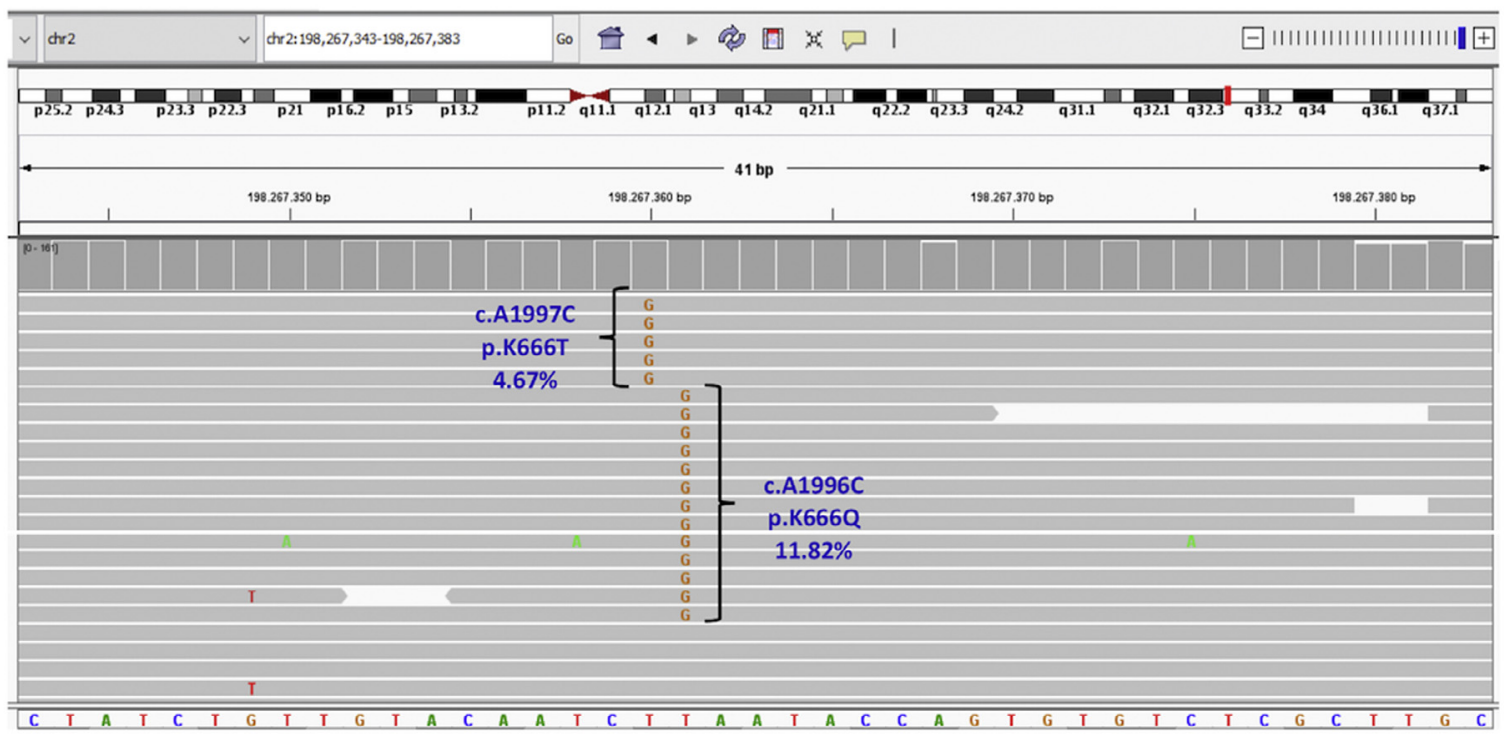

SF3B1

Figure 1. (A) Molecular background in chronic lymphocytic leukemia (CLL) patients with chromosomal gains. Mutations and chromosomal alteration information are shown across the list of genes or fluorescence in situ hybridization (FISH) abnormalities (rows) for seven CLL samples that underwent next-generation sequencing (columns). The number of chromosomes with the FISH panel (percentage of cells with the gain) was detailed in the last row for each case. (B) Exemplary screenshot from the Integrative Genomics Viewer revealing the reads obtained within chromosome region chr2: 198267343-198267383 in patient ID3. Two SF3B1 sequence variants are depicted that are located on distinct sequencing reads, indicating the presence of two individual mutational clones in this patient. 
The remaining genes with mutations were NOTCH1, RPS15, MGA, PDCH10, FUBP1, IGLL5, and KLHL6, which were mutated in one patient each $(1 / 7,14.3 \%)$. The incidence of mutations of these CLL drivers was higher in this cohort of CLL with chromosomal gains than in the reported CLL series [7,8,11,12]. Specifically, BRAF mutations were approximately 10-fold more frequent in hyperdiploid CLLs than in other cohorts $[7,8,13]$. Of note, these $2 B R A F$ mutations were located in the same codon (p.D594N y p.D594G) in two cases, not involving the canonical hotspot (V600E) seen in other malignancies $[14,15]$. Sixteen of the total of 21 mutations were previously reported in the Catalogue of Somatic Mutations in Cancer (COSMIC) or in other CLL sequencing studies [7,8] (Supplementary Table E5).

It should be noted that $61.9 \%$ of the mutations (13/21) were detected in genes previously associated with a poor prognosis in CLL [7,8], such as ATM $(n=3), T P 53(n=3)$, SF3B1 $(n=3)$, BRAF $(n=2)$, NOTCHI $(n=1)$, and RPS15 $(n=1)$. By contrast, none of the patients exhibited MYD88 mutations, which have been associated with a good prognosis in CLL [16].

Most of the patients $(6 / 7,85.7 \%)$ had more than 2 CLL driver mutations within the set of genes analyzed in our study. Patient P2, who was analyzed at the time of CLL progression, had the largest number of mutations: 6. Of note, this patient exhibited 2 different mutations on SF3BI affecting the same codon but in different clones (Figure 1B). This clearly illustrates intratumoral genetic heterogeneity, which could be a reflex of the presence of genomic instability $[7,17]$.

\section{Most CLL patients with chromosomal gains exhibited genetic alterations in genes involved in the DNA damage response}

Interestingly, $71.4 \%$ of the patients (5/7) exhibited alterations in TP53 or ATM (deletion and/or mutation). Mutational inactivation of these genes involved in DDR mechanisms is an established hallmark of CLL and is associated with high genomic instability $[18,19]$. Three patients had TP53 alterations: only mutation (P7) and mutation plus $17 \mathrm{p}-(\mathrm{P} 2$ and $\mathrm{P} 3)$. One of the patients (P2) also had a mutation on RPS15, a gene recently associated with TP53 alterations [20]. Of note, two TP53-mutated patients (P2 and P7) also had SF3B1 mutations, which have been associated with an altered DDR [21,22]. Two patients harbored ATM alterations: only mutation (P1) and mutation plus 11q(P5). Both patients also had BRAF mutations, suggesting that the combination of these two mutated genes $(A T M+B R A F)$ could be related to hyperdiploid karyotype. Normal BCR and pre-BCR signaling occurs through $B R A F$, which activates ERK proteins [22], resulting in the deregulation of a set of genes. BRAF mutations target amino acids located in the P-loop of the kinase, resulting in a weaker activation than the canonical V600E [22].

Comparison of the group of CLL patients with hyperdiploidy and the cohort of CLL patients without hyperdiploidy revealed that the frequency of mutations in TP53 or $A T M$ was significantly higher in patients with hyperdiploidy (72\% vs. $12 \%, P=0.002)$. Mutations in these genes could impair DDR mechanisms, which could result in genetic instability in CLL patients [21,23]. Notably, a larger number of mutations were observed in CLL patients with hyperdiploidy than in those without this genetic background (median number of mutations $=4$ vs. $1, P=0.001$ ) In addition, within patients with hyperdiploidy, the percentage of cases with more than one mutated gene was higher than that in the control group (86\% vs. $20 \%, P=0.001)$. Supplementary Table E5 lists the mutations in the CLL patients sequenced in this study. Functional studies should be performed to confirm an impairment of the DDR in CLL primary cells in patients with hyperdiploidy.

In conclusion, the presence of mutations in CLL driver genes and high-risk genetic alterations involving the DDR pathway is greatly enriched in CLL patients with several chromosomal gains, suggesting that genomic instability could be a hallmark of hyperdiploidy in CLL.

\section{Acknowledgments}

This work was supported by grants from the Spanish Fondo de Investigaciones Sanitarias (PI15/01471, PI18/ 01500); by the Instituto de Salud Carlos III (ISCIII), European Regional Development Fund (ERDF) "Una manera de hacer Europa"; and by grants from Red Temática de Investigación Cooperativa en Cáncer (RTICC) and Centro de Investigación Biomédica en Red de Cáncer (CIBERONC) (RD12/0036/0069). MH-S is supported by FEHHJanssen ("Sociedad Española de Hematología y Hemoterapia"). AER-V and JMH-S are supported by a research grant from FEHH (Fundación Española de Hematología y Hemoterapia). MQ-Á is supported by a grant from "Ayuda Predoctoral de la Junta de Castilla y León" (JCYL-EDU/529/2017).

\section{Conflict of Interest Disclosure}

The authors declare no competing interests regarding the publication of this article.

\section{References}

1. Moorman AV, Harrison CJ, Buck GA, et al. Karyotype is an independent prognostic factor in adult acute lymphoblastic leukemia (ALL): analysis of cytogenetic data from patients treated on the Medical Research Council (MRC) UKALLXII/Eastern Cooperative Oncology Group (ECOG) 2993 trial. Blood. 2007; 109:3189-3197.

2. Wuilleme S, Robillard N, Lode L, et al. Ploidy, as detected by fluorescence in situ hybridization, defines different subgroups in multiple myeloma. Leukemia. 2005;19:275-278.

3. DeNicola M, Pullarkat S, Yea S, Rao N, Yang L, Tirado CA. Hyperdiploidy in CLL/SLL: A rare cytogenetic event associated 
with poor prognosis. J Am Assoc Gene Technologists. 2014;40: 22-24.

4. Gonzalez-Gascon YMI, Martin AA, Hernandez-Sanchez M, et al. Hyperdiploidy as a rare event that accompanies poor prognosis markers in CLL. Eur J Haematol. 2017;98:142-148.

5. Vundinti BR, Korgaonkar S, Kerketta L, Ghosh K. A rare case of B-cell chronic lymphocytic leukemia with $\mathrm{t}(2 ; 14)(\mathrm{p} 13 ; \mathrm{q} 32)$, $+\mathrm{X},+11,+12,+13,+\operatorname{der}(1 \mathrm{p})$ karyotype. Indian J Cancer. 2011; 48:387-388.

6. Watanabe A, Inokuchi K, Yamaguchi $\mathrm{H}$, et al. Near-triploidy and near-tetraploidy in hematological malignancies and mutation of the p53 gene. Clin Lab Haematol. 2004;26:25-30.

7. Landau DA, Tausch E, Taylor-Weiner AN, et al. Mutations driving CLL and their evolution in progression and relapse. Nature. 2015;526:525-530.

8. Puente XS, Bea S, Valdes-Mas R, et al. Non-coding recurrent mutations in chronic lymphocytic leukaemia. Nature. 2015;526:510-524.

9. DePristo MA, Banks E, Poplin R, et al. A framework for variation discovery and genotyping using next-generation DNA sequencing data. Nat Genet. 2011;43:491-498.

10. Li H, Durbin R. Fast and accurate long-read alignment with Burrows-Wheeler transform. Bioinformatics. 2010;26:589-595.

11. Puente XS, Pinyol M, Quesada V, et al. Whole-genome sequencing identifies recurrent mutations in chronic lymphocytic leukaemia. Nature. 2011;475:101-105.

12. Quesada V, Conde L, Villamor N, et al. Exome sequencing identifies recurrent mutations of the splicing factor SF3B1 gene in chronic lymphocytic leukemia. Nat Genet. 2012;44:47-52.

13. Jebaraj BM, Kienle D, Buhler A, et al. BRAF mutations in chronic lymphocytic leukemia. Leuk Lymphoma. 2013;54:1177-1182.
14. Brastianos PK, Taylor-Weiner A, Manley PE, et al. Exome sequencing identifies BRAF mutations in papillary craniopharyngiomas. Nat Genet. 2014;46:161-165.

15. Tiacci E, Trifonov V, Schiavoni G, et al. BRAF mutations in hairy-cell leukemia. The N Engl J Med. 2011;364:2305-2315.

16. Martinez-Trillos A, Pinyol M, Navarro A, et al. Mutations in TLR/MYD88 pathway identify a subset of young chronic lymphocytic leukemia patients with favorable outcome. Blood. 2014; 123:3790-3796.

17. Landau DA, Carter SL, Stojanov P, et al. Evolution and impact of subclonal mutations in chronic lymphocytic leukemia. Cell. 2013;152:714-726

18. Reinhardt HC, Schumacher B. The p53 network: Cellular and systemic DNA damage responses in aging and cancer. Trends Genet. 2012;28:128-136.

19. Stankovic T, Skowronska A. The role of ATM mutations and $11 \mathrm{q}$ deletions in disease progression in chronic lymphocytic leukemia. Leuk Lymphoma. 2014;55:1227-1239.

20. Yu L, Kim HT, Kasar S, et al. Survival of Del17p CLL depends on genomic complexity and somatic mutation. Clin Cancer Res. 2017;23:735-745

21. Te Raa GD, Derks IA, Navrkalova V, et al. The impact of SF3B1 mutations in CLL on the DNA-damage response. Leukemia. 2015;29:1133-1142.

22. Wang L, Brooks AN, Fan J, et al. Transcriptomic characterization of SF3B1 mutation reveals its pleiotropic effects in chronic lymphocytic leukemia. Cancer Cell. 2016;30:750-763.

23. Mohr J, Helfrich H, Fuge M, et al. DNA damage-induced transcriptional program in CLL: biological and diagnostic implications for functional p53 testing. Blood. 2011;117:1622-1632. 